\title{
On the detection of satellites of extrasolar planets with the method of transits
}

\author{
P. Sartoretti ${ }^{1}$ and J. Schneider ${ }^{2}$ \\ 1 Institut d'Astrophysique de Paris, 98 bis boulevard Arago, 75014 Paris, France \\ 2 DARC, Observatoire de Meudon, Place Jules Janssen, 92195 Meudon, France
}

Received March 27; accepted September 9, 1998

\begin{abstract}
We compute the detection probability of satellites of extrasolar planets with the method of transits, under the assumption that the duration of the observations is at least as long as the planet orbital period. We separate the cases when the parent planet does and does not also transit over the star. The possible satellites are assumed to have orbital radii between the Roche limit and the Hill radius. We find that if a satellite is extended enough to produce a detectable drop in the stellar lightcurve, the probability to detect it when the planet also transits over the star is nearly unity. If the planet does not transit, the probability to detect a Jupiter-like or terrestrial satellite is modest, but it can be comparable to that of detecting a planet if the satellite orbital radius is large. If a satellite is not extended enough to produce a detectable drop in the stellar lightcurve during a transit, it might still be detected through the time shift of planetary transits resulting from the rotation of the planet around the barycenter of the planet-satellite system.
\end{abstract}

Key words: planets and satellites: general — stars: planetary systems — techniques: photometric

\section{Introduction}

Struve (1952) first proposed to search for extrasolar planets using the method of transits. The method consists in monitoring photometrically a star to eventually detect a luminosity drop caused by the transit of a planet across the line of sight. The photometric drop is proportional to the squared ratio of the planet to stellar radii, and hence, it requires high precision photometry to be detected. An extended planet like Jupiter, for example, would produce

Send offprint requests to: P. Sartoretti

e-mail: sartoret@iap.fr a photometric drop corresponding to only $\sim 1 \%$ of the solar luminosity. Since the observations should be at least as long as a planet period to detect a transit, searches for long-period planets similar to those in our solar system require time consuming observations. In addition, longperiod planets have a large orbital radii, and hence, a weak probability to present the right line-of-sight inclination of the orbital plane that will produce an observable transit. Therefore, thousands of stars should be observed for several years with high precision photometry to exploit the method of transits. These constraints have seriously limited the applications of the method after it was first proposed.

Searches for extrasolar planets based on the method of transit are now starting in earnest after the discovery of Jupiter-like planets, with orbital periods much shorter than that of Jupiter, around solar-type stars (Mayor \& Queloz 1995; Marcy et al. 1996; Butler et al. 1997; Cochran et al. 1997). A network was organized to search for transit of extrasolar planets (the "TEP" network). Moreover, space missions have been proposed to conduct long and continuous observations of large samples of stars to exploit the method of transits (Koch et al. 1996; Borucki et al. 1997). One of the main programs of the satellite COROT, that will be launched in 2001, is to achieve this goal (Schneider et al. 1997).

Soon, therefore, we expect to obtain stellar lightcurves showing possible transits of extrasolar planets. Some of these planets will have satellites, that may also transit over the star and be detectable. The expectation rate of planetary transits relies on models of the formation and evolution of planetary systems. Little is known on the distributions in number, size, orbital radius of extrasolar planets. Moreover, the absolute rate of occurence of Jupiter-like planets and the relative occurence of Jupiter-like with respect to Earth-like planets are unknown. Jupiter-like planets should produce stronger transit signals because terrestrial planets are significantly smaller, the expected maximum radius being less than about 2.5 times the Earth 
radius (Guillot et al. 1996). In this context, the possibility for Jupiter-like planets to have terrestrial satellites raises a crucial issue about the possibility to detect Earth-like objects outside our solar system. A satellite should imprint characteristic signatures on the transit lightcurve of a planet. In addition, the presence of satellites may increase the overall probability of detecting transits of extrasolar planets. The detection of planetary satellites with the method of transits, however, has never been investigated.

In this paper we analyze the probability of detecting satellites of extrasolar planets with the method of transits. Detection rates are computed under the assumption that a planet-satellite system orbits around the target star, and that the duration of the observations is at least as long as the planet orbital period. In Sect. 2 below we compute the probability to detect directly a satellite transit (i.e., the satellite is extended enough to produce a detectable minimum in the lightcurve), separating the cases when the parent planet does and does not also transit over the star. We present the results of this analysis in Sect. 3 and show examples of transit lightcurves revealing the presence of Jupiter-like and terrestrial satellites. Finally, in Sect. 4 we describe the case in which the satellite is too small to produce a detectable minimum in the lightcurve, but its presence may still be inferred indirectly by the timing of the planetary transits.

\section{Direct photometric detection: Probability}

We first compute the probability of detecting the transit of a planetary satellite in front of the parent star. We start by computing the luminosity drop $\Delta F_{*}$ produced by an object, planet or satellite of a planet, on the line of sight to a star of luminosity $F_{*}$. This can be expressed as $\Delta F_{*}=\pi r_{0}^{2} I$, where $r_{0}$ is the radius of the object, and $I$ is the surface brightness of the star where the object is producing the occultation. We assume that the star is spherical and that the radial dependence of the surface brightness can be described by the standard limb-darkening law $I(r)=I_{0}\left(1-\mu+\mu \sqrt{1-\left(r / r_{*}\right)^{2}}\right)$. Here, $r$ is the projected distance from the star center to the object, $r_{*}$ is the radius of the star, and $\mu$ is the limb-darkening parameter that depends on wavelength. For reference, in the visible a solar-type star has $\mu \approx 0.5$ (Allen 1973, p. 171). Since $F_{*}$ is the integral of $I(r)$ over the star's area, we can write the relative drop in luminosity imprinted by the object as:

$\Delta F_{*} / F_{*}=\left(r_{0} / r_{*}\right)^{2} \times \frac{1-\mu+\mu \sqrt{1-\left(r / r_{*}\right)^{2}}}{1-\mu / 3}$.

This expression reduces to $\Delta F_{*} / F_{*}=\left(r_{0} / r_{*}\right)^{2}$ when $r=$ $0.75 r_{*}$.

Numerical evaluations of Eq. (1) show that the drop in luminosity caused by an occulting object depends only weakly on the projected distance of the object to the star center, except when the object is very close to the limb. For example, adopting $\mu=0.5$, Eq. (1) indicates that $\Delta F_{*} / F_{*}$ is only $30 \%$ smaller for $r=0.9 r_{*}$ than for $r=0$. In the following, therefore, when computing detection probabilities for planets and satellites of planets transiting in front of a parent star, we consider all projected trajectories with $r \leq r_{*}$. As Eq. (1) shows, $\Delta F_{*} / F_{*}$ is roughly of the order of $\left(r_{0} / r_{*}\right)^{2}$, and for a Jupiter-like or Earth-like planet occulting a solar-type star this implies $\Delta F_{*} / F_{*} \approx 10^{-2}$ or $10^{-4}$, respectively, at $r \lesssim r_{*}$. For reference, COROT is expected to detect planets or satellites with $r_{0} \gtrsim 2 r_{\mathrm{E}}$, where $r_{\mathrm{E}}$ is the Earth radius.

We do not speculate here on the origin of satellites with radii $r_{0} \gtrsim 2 r_{\mathrm{E}}$ that could be detected with COROT. Their formation could be associated to that of giant planets, or they could form or be capured ulteriorly. We note, however, that the lifetimes of such satellites before they collapse on their parent planets are expected to be relatively long because of the high tidal dissipation factor of Jupiter-like planets $\left(Q_{\mathrm{J}} \sim 10^{5}\right.$; see, e.g., Burns 1986). In fact, based on the calculations by Burns, the expected lifetime of a satellite with $r_{0} \sim 2 r_{\mathrm{E}}$ at a distance $\gtrsim 10 r_{\mathrm{J}}$ from a Jupiter-like planet with orbital period $\gtrsim 20$ days is $\gtrsim 10^{9} \mathrm{yr}$ (where $r_{\mathrm{J}} \sim 11 r_{\mathrm{E}}$ is the Jupiter radius). As mentioned in Sect. 1, the expected occurence of Jupiterlike planets is independent of orbital radius. It is conceivable, therefore, that extended satellites be found around Jupiter-like planets in relatively close orbit (i.e., with short periods) around solar-type stars.

Stellar variability, unless it is much stronger than that exhibited by the Sun, should not prevent the detection of transits of even Earth-like planets. In fact, the Sun variability is $\sim 10^{-5}$ on a time scale of several hours, as measured by the Solar Maximum Mission satellite, the Upper Atmosphere Research Satellite, and the Solar and Heliospheric Observatory for the Sun (Fröhlich 1987; Willson \& Hudson 1991). The photometric variability due to sunspot activity may be more important, but it should not be confused with planetary transits because it depends on wavelength and is not periodic.

We now compute the probability for the transit of a planetary satellite to be detected. In this section the satellite is assumed to be extended enough to produce a detectable minimum in the lightcurve. The detection probability is given by the product of two independent probabilities, which we define as "geometric probability" and "orbital probability". In the remainder of this paper, we express all distances in units of $r_{*}=1$ and all masses in units of a solar mass.

\subsection{Geometric probability}

The geometric probability is the probability for the planetsatellite system to have a favorable orbital inclination angle so that the projected satellite orbit can intersect the star. We first describe the geometric conditions required 
for the transit of a planet and then extend the results to the case in which the planet has a satellite. We assume throughout this paper that the orbits are keplerian and circular. A transit of a planet is geometrically possible when the projected orbit intersects the star. The projected position of the planet with respect to the star center can be most generally parameterized as:

$y_{\mathrm{p}}=a_{\mathrm{p}}\left(\cos \nu \sin \Omega_{\mathrm{p}}+\sin \nu \cos \Omega_{\mathrm{p}} \cos i_{\mathrm{p}}\right)$

$z_{\mathrm{p}}=a_{\mathrm{p}} \sin \nu \sin i_{\mathrm{p}}$

where $a_{\mathrm{p}}$ is the planet orbital radius, $i_{\mathrm{p}}$ is the line-of-sight inclination of the orbital plane, $\Omega_{\mathrm{p}}$ is the longitude of the ascending node, and $\nu$ is the planet phase angle on the orbit. A transit then takes place when $\sqrt{y_{\mathrm{p}}^{2}+z_{\mathrm{p}}^{2}} \leq 1$.

The geometric probability for a transit of the planet can be estimated by noticing that it corresponds to all line-of-sight inclinations of the planetary orbital plane less than the critical angle $\sin i_{\mathrm{p} 0} \approx i_{\mathrm{p} 0}=1 / a_{\mathrm{p}}$, where again $a_{\mathrm{p}}$ is expressed in units of the stellar radius. Therefore, the total geometric probability of a planetary transit is given by the ratio of the area of detectability, $2 \times 2 \pi a_{\mathrm{p}}$, to the entire possible area covered by planetary orbits, $4 \pi a_{\mathrm{p}}^{2}$. This is $a_{\mathrm{p}}^{-1}$, or $i_{\mathrm{p} 0}$. The differential geometric probability that a planet transit occurs with orbital inclination angle between $i_{\mathrm{p}}$ and $i_{\mathrm{p}}+\mathrm{d} i_{\mathrm{p}}$ can then be written as:

$$
\begin{array}{ll}
P_{\mathrm{g}}\left(i_{\mathrm{p}}\right) \mathrm{d} i_{\mathrm{p}}=\mathrm{d} i_{\mathrm{p}} & \text { for } 0 \leq i_{\mathrm{p}} \leq i_{\mathrm{p} 0} \\
P_{\mathrm{g}}\left(i_{\mathrm{p}}\right) \mathrm{d} i_{\mathrm{p}}=0 & \text { for } i_{\mathrm{p} 0} \leq i_{\mathrm{p}} \leq \pi / 2,
\end{array}
$$

with

$i_{\mathrm{p} 0}=a_{\mathrm{p}}^{-1}$. We now extend this reasoning to the case in which the planet has a satellite. We denote by $a_{\mathrm{s}}$ the satellite orbital radius around the planet and by $i_{\mathrm{ps}}$ the angle between the planet and satellite orbital planes. If any value of $i_{\mathrm{ps}}$ is allowed, by analogy with the above argument the total geometric probability for the planet or satellite to transit in front of the star will be $a_{\mathrm{p}}^{-1}\left(1+a_{\mathrm{s}}\right)$. Satellites of the Solar system, however, have orbital planes inclined by a few degrees at most with respect to that of their parent planet. Hence, we fix $i_{\mathrm{ps}}$, which has for effect to limit the total probability for a planetary or satellite transit to $a_{\mathrm{p}}^{-1}\left(1+a_{\mathrm{s}} \sin i_{\mathrm{s} 1}\right) \approx a_{\mathrm{p}}^{-1}\left(1+a_{\mathrm{s}} i_{\mathrm{s} 1}\right)$. In this expression, $i_{\mathrm{s} 1}=\left(1+a_{\mathrm{p}} i_{\mathrm{ps}}\right)\left(a_{\mathrm{p}}-a_{\mathrm{s}}\right)^{-1}$ is the maximum allowed lineof-sight inclination of the satellite orbital plane, that is determined from the condition $i_{\mathrm{s} 1}=i_{\mathrm{ps}}+a_{\mathrm{p}}^{-1}\left(1+a_{\mathrm{s}} i_{\mathrm{s} 1}\right)$. Consequently, the maximum line-of-sight inclination of the planetary orbital plane leading to a transit of the satellite is $i_{\mathrm{p} 1}=i_{\mathrm{s} 1}-i_{\mathrm{ps}}=\left(1+a_{\mathrm{s}} i_{\mathrm{ps}}\right)\left(a_{\mathrm{p}}-a_{\mathrm{s}}\right)^{-1}$.

We can reexpress $a_{\mathrm{s}}$ in terms of the planetary orbital radius, $a_{\mathrm{p}}$. The satellite orbital radius must satisfy the condition $a_{\mathrm{R}}<a_{\mathrm{s}}<a_{\mathrm{H}}$, where $a_{\mathrm{R}}$ and $a_{\mathrm{H}}$ are the planet Roche and Hill radii, respectively. We parameterize $a_{\mathrm{s}}$ in terms of the Hill radius as $a_{\mathrm{s}}=\xi^{-1} a_{\mathrm{H}}=\xi^{-1} a_{\mathrm{p}} M_{\mathrm{p}}^{1 / 3}$, where $M_{\mathrm{p}}$ is the mass of the planet in units of a solar mass, with $1 \leq \xi \leq a_{\mathrm{H}} / a_{\mathrm{R}}$. We further adopt the notation
$X=\xi^{-1} M_{\mathrm{p}}^{1 / 3}$. With these notations, the maximum lineof-sight inclination of the planetary orbital plane leading to a transit of the satellite, $i_{\mathrm{p} 1}$, can be rewritten in terms of $a_{\mathrm{p}}, X$ and $i_{\mathrm{ps}}$. The differential geometric probability that a satellite transit occurs when the planetary orbital inclination angle ranges between $i_{\mathrm{p}}$ and $i_{\mathrm{p}}+\mathrm{d} i_{\mathrm{p}}$ is thus:

$$
\begin{array}{ll}
P_{\mathrm{g}}\left(i_{\mathrm{p}}\right) \mathrm{d} i_{\mathrm{p}}=\mathrm{d} i_{\mathrm{p}} & \text { for } 0 \leq i_{\mathrm{p}} \leq i_{\mathrm{p} 1} \\
P_{\mathrm{g}}\left(i_{\mathrm{p}}\right) \mathrm{d} i_{\mathrm{p}}=0 & \text { for } i_{\mathrm{p} 1} \leq i_{\mathrm{p}} \leq \pi / 2 .
\end{array}
$$

with

$i_{\mathrm{p} 1}=\left(1+a_{\mathrm{p}} X i_{\mathrm{ps}}\right)\left[a_{\mathrm{p}}(1-X)\right]^{-1}$.

Equations (4) and (7) then show immediately that for lineof-sight inclinations of the planetary orbital plane in the range $\left[i_{\mathrm{p} 0}, i_{\mathrm{p} 1}\right]$, only the satellite can transit in front of the star.

In the remainder of this paper, all expressions are computed by adopting the above parameterization of $a_{\mathrm{s}}$ in terms of $a_{\mathrm{p}}$ via the parameter $\xi$, that allows us to sample all possible satellite orbital radii. The results can be reformulated straightforwardly in the context of any alternative parameterization using $X=\xi^{-1} M_{\mathrm{p}}^{1 / 3}=a_{\mathrm{s}} a_{\mathrm{p}}^{-1}$.

\subsection{Orbital probability}

For a satellite transit to occur, the satellite must be in the part of its orbit intersecting the star when the planet is in a favorable location. This can be described by an orbital probability function. We assume that the duration of the observations is at least as long as the planetary orbital period. Therefore, the planet will always sample the part of its orbit that is favorable for a satellite transit if the condition $i_{\mathrm{p}} \leq i_{\mathrm{p} 1}$ is satisfied. To express the orbital probability function of the satellite, it is convenient to introduce the variable

$\tilde{a}=a_{\mathrm{s}} i_{\mathrm{s}}-a_{\mathrm{p}} i_{\mathrm{p}}+1=a_{\mathrm{p}} i_{\mathrm{p}}(X-1)+1+X a_{\mathrm{p}} i_{\mathrm{ps}}$,

which measures the projected distance from the stellar limb to the point of intersection of the satellite's orbit with the planet-star axis at the time when the planet projects closest to the star center. For a satellite transit to occur one must have $\tilde{a}>0$, which is equivalent to the condition $i_{\mathrm{p}}<i_{\mathrm{p} 1}$.

Three limiting conditions characterize the orbital probability associated to a geometrically possible transit $(\tilde{a}>0)$. First, the planet itself is transiting, and at a given time the satellite's orbit projects entirely on the stellar area. This corresponds to $\tilde{a}>2 a_{\mathrm{s}} i_{\mathrm{s}}$, and in this case the orbital probability is unity. Second, at any given time the satellite's orbit projects only partially on the star area. In this case the orbital probability depends on the comparison between $\tilde{a}$ and the stellar diameter. If $\tilde{a}<2$, the orbital probability, i.e., the fraction of the satellite's orbit which will intersect the star as the planet orbits is $\tilde{a}\left(2 a_{\mathrm{s}} i_{\mathrm{s}}\right)^{-1}$. If, on the other hand, $\tilde{a}>2$, then the orbital probability reduces to $2 \times\left(2 a_{\mathrm{s}} i_{\mathrm{s}}\right)^{-1}=\left(a_{\mathrm{s}} i_{\mathrm{s}}\right)^{-1}$. 
The above limiting conditions on $\tilde{a}$ translate via Eq. (10) into conditions on the line-of-sight inclination of the planetary orbital plane, $i_{\mathrm{p}}$, for given $a_{\mathrm{p}}, X$ and $i_{\mathrm{ps}}$. The orbital probability of a geometrically possible satellite transit is therefore given as a function of $i_{\mathrm{p}}$ by:

$P_{\mathrm{o}}\left(i_{\mathrm{p}}\right)=1$ for $i_{\mathrm{p}} \leq i_{\mathrm{p} 2}$

$P_{\mathrm{o}}\left(i_{\mathrm{p}}\right)=\tilde{a}\left(2 a_{\mathrm{s}} i_{\mathrm{s}}\right)^{-1}$ for $i_{\mathrm{p}} \geq i_{\mathrm{p} 2}$ and $i_{\mathrm{p}} \geq i_{\mathrm{p} 3}$

$P_{\mathrm{o}}\left(i_{\mathrm{p}}\right)=\left(a_{\mathrm{s}} i_{\mathrm{s}}\right)^{-1}$ for $i_{\mathrm{p}} \geq i_{\mathrm{p} 2}$ and $i_{\mathrm{p}} \leq i_{\mathrm{p} 3}$,

with

$i_{\mathrm{p} 2}=\left(1-X a_{\mathrm{p}} i_{\mathrm{ps}}\right)\left[a_{\mathrm{p}}(1+X)\right]^{-1}$

$i_{\mathrm{p} 3}=\left(X a_{\mathrm{p}} i_{\mathrm{ps}}-1\right)\left[a_{\mathrm{p}}(1-X)\right]^{-1}$.

Equations (11)-(13) can also be rewritten in terms of $i_{\mathrm{p}}$, $a_{\mathrm{p}}, X$ and $i_{\mathrm{ps}}$. If the duration of the observations is $n_{\mathrm{t}}$ times longer than the planet period, the orbital probability must be multiplied by $n_{\mathrm{t}}$, until eventually one reaches $P_{\mathrm{o}}=1$.

\subsection{Detection probability}

The detection probability of the satellite is the product of the geometric and orbital probabilities. We let the planetary orbital radius $a_{\mathrm{p}}$ vary and fix the the angle between the planet and satellite orbital planes, $i_{\mathrm{ps}}$, and the parameter $X$. Using the same notations as above, the probability to detect a satellite transit is therefore

$P_{\text {det }}\left(a_{\mathrm{p}}\right)=\int_{0}^{i_{\mathrm{p} 1}} \mathrm{~d} i_{\mathrm{p}} P_{\mathrm{o}}\left(i_{\mathrm{p}}\right)$

while the probability to detect a satellite transit without a transit of the planet is

$P_{\mathrm{det}}^{\mathrm{S}}\left(a_{\mathrm{p}}\right)=\int_{i_{\mathrm{p} 0}}^{i_{\mathrm{p} 1}} \mathrm{~d} i_{\mathrm{p}} P_{\mathrm{o}}\left(i_{\mathrm{p}}\right)$.

Also of interest is the probability to detect a transit of the satellite when the planet is itself assumed to transit. This is given by

$P_{\text {det }}^{\mathrm{P}}\left(a_{\mathrm{p}}\right)=a_{\mathrm{p}} \int_{0}^{i_{\mathrm{p} 0}} \mathrm{~d} i_{\mathrm{p}} P_{\mathrm{o}}\left(i_{\mathrm{p}}\right)$,

since the probability for a transit of the planet is $a_{\mathrm{p}}^{-1}$.

As noted in Sect. 2.2, the form of the integrand in Eqs. (16) - (18) depends on the value of $i_{\mathrm{p}}$ relative to $i_{2}$ and $i_{3}$. For fixed $i_{\mathrm{ps}}$ and $X$, this depends on the parameter $a_{\mathrm{p}}$. More specifically, if $a_{1}=\left(X i_{\mathrm{ps}}\right)^{-1}$ and $a_{2}=$ $(2-X)\left(X i_{\mathrm{ps}}\right)^{-1}$, then $P_{\mathrm{det}}\left(a_{\mathrm{p}}\right), P_{\mathrm{det}}^{\mathrm{S}}\left(a_{\mathrm{p}}\right)$ and $P_{\mathrm{det}}^{\mathrm{P}}\left(a_{\mathrm{p}}\right)$ can be computed by breaking the integrals in Eqs. (16)-(18) according to the rule

$i_{\mathrm{p} 3}<0<i_{\mathrm{p} 2}<i_{\mathrm{p} 0}<i_{\mathrm{p} 1}$

$$
\begin{aligned}
& \text { for } a_{\mathrm{p}}<a_{1}, \\
& \text { for } a_{1}<a_{\mathrm{p}}<a_{2}, \\
& \text { for } a_{2}<a_{\mathrm{p}} .
\end{aligned}
$$$$
i_{\mathrm{p} 2}<0<i_{\mathrm{p} 0}<i_{\mathrm{p} 3}<i_{\mathrm{p} 1}
$$

For the values of $X$ under consideration, $a_{1}$ is always smaller than $a_{2}$.

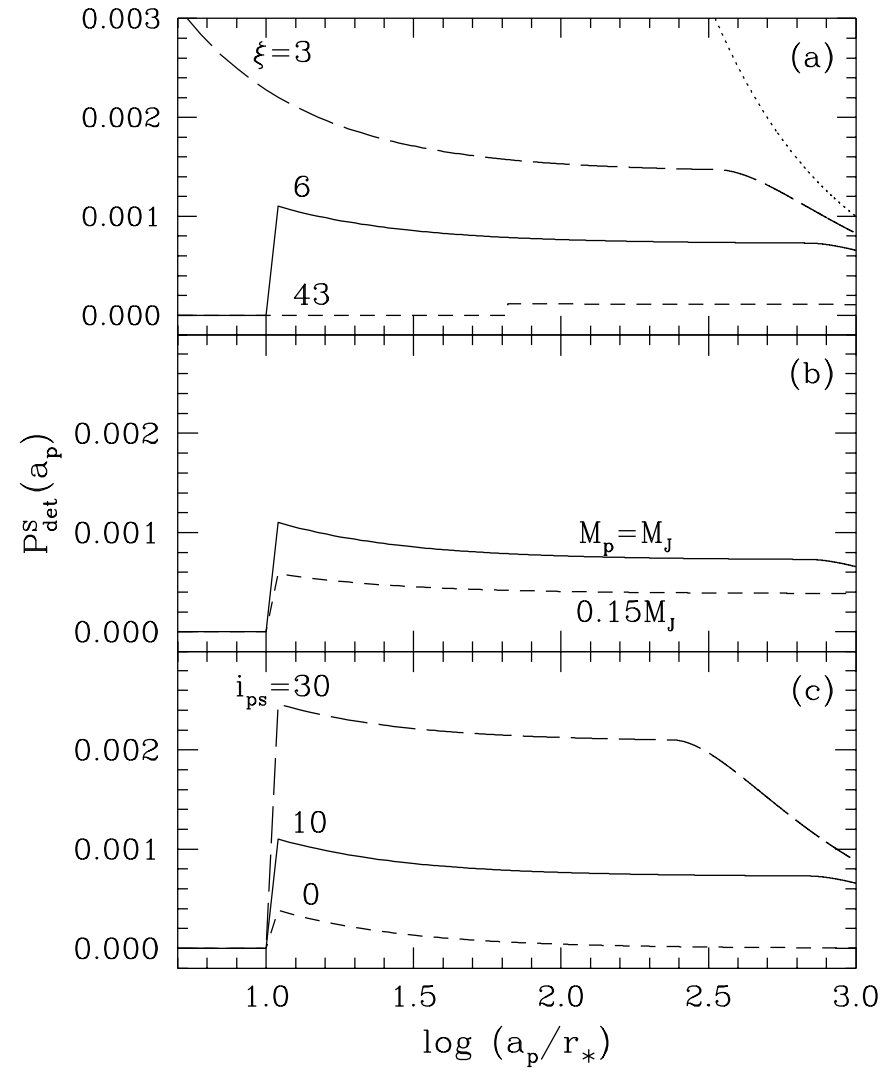

Fig. 1. Probability $P_{\text {det }}^{\mathrm{S}}\left(a_{\mathrm{p}}\right)$ of detecting a satellite transit during a planetary period, when the parent planet does not itself transit over the star. a) For different values of $\xi=M_{\mathrm{p}}^{1 / 3} a_{\mathrm{p}} a_{\mathrm{s}}^{-1}$, as indicated, at fixed angle $i_{\mathrm{ps}}=10^{\circ}$ between the planet and satellite orbital planes and fixed planetary mass $M_{\mathrm{p}}=M_{\mathrm{J}}\left(a_{\mathrm{p}}\right.$ and $a_{\mathrm{s}}$ are, respectively, the planet and satellite orbital radii). b) For two values of $M_{\mathrm{p}}$, as indicated, at fixed $\xi=6$ and $i_{\mathrm{ps}}=10^{\circ}$. c) For different values of $i_{\mathrm{ps}}$, as indicated, at fixed $\xi=6$ and $M_{\mathrm{p}}=M_{\mathrm{J}}$

\section{Direct photometric detection: Results}

Figure 1 shows $P_{\text {det }}^{\mathrm{S}}\left(a_{\mathrm{p}}\right)$, the probability to detect a satellite transit without a transit of the parent planet, for various assumptions on the planet and satellite parameters. The probability is computed assuming that the duration of the observations is always a planetary period $\left(n_{\mathrm{t}}=1\right)$. In Fig. 1a we show the effect of varying the parameter $\xi$ that controls the satellite orbital radius, for fixed planet mass $M_{\mathrm{p}}=M_{\mathrm{J}}$ (where $M_{\mathrm{J}}$ is the Jupiter mass) and angle between the satellite and planet orbital planes $i_{\mathrm{ps}}=10^{\circ}$. For a given $\xi, P_{\mathrm{det}}^{\mathrm{S}}\left(a_{\mathrm{p}}\right)$ decreases with increasing $a_{\mathrm{p}}$. The reason for this is that the range of favorable line-of-sight inclinations of the planet orbital plane, $i_{\mathrm{p} 1}-i_{\mathrm{p} 0}$, becomes narrower. The drop is more pronounced when $a_{\mathrm{p}}$ is small because then, $i_{\mathrm{p} 1}-i_{\mathrm{p} 0}$ is roughly proportional to $a_{\mathrm{p}}^{-1}$. For large $a_{\mathrm{p}}, i_{\mathrm{p} 1}-i_{\mathrm{p} 0}$ reaches the asymptotic value $X i_{\mathrm{ps}}(1-X)^{-1}$, and $P_{\mathrm{det}}^{\mathrm{S}}\left(a_{\mathrm{p}}\right)$ becomes nearly constant. When the planet orbital radius reaches the value $a_{\mathrm{p}}=a_{2}$, however, $P_{\text {det }}^{\mathrm{S}}\left(a_{\mathrm{p}}\right)$ undergoes a 


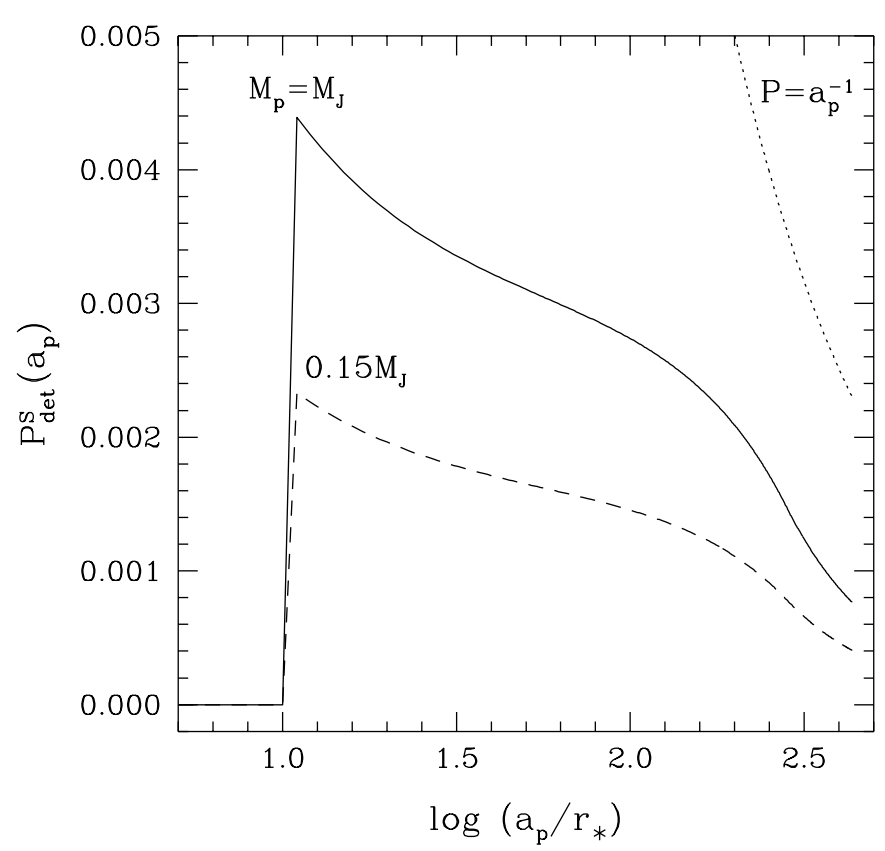

Fig. 2. Probability $P_{\mathrm{det}}^{\mathrm{S}}\left(a_{\mathrm{p}}\right)$ of detecting a satellite transit during a period of observations of 3 years, when the parent planet does not itself transit over the star. Two values of $M_{\mathrm{p}}$ are considered, as indicated, at fixed $\xi=6$ and $i_{\mathrm{ps}}=10^{\circ}$ (as in Fig. 1b). The dotted line shows the probability $P=a^{-1}$ of detecting a planet

new drop as the orbital probability of catching the satellite decreases (see Eqs. (12), (13) and (21)). This drop occurs at $\log \left(a_{\mathrm{p}}\right) \gtrsim 2.6$ for $\xi=3$ and at larger $a_{\mathrm{p}}$ for $\xi=6$ and 43.

Figure 1a shows that the detection probability is higher for satellites with smaller $\xi$, corresponding to larger orbital radii, because the geometric probability that defines the upper limit $i_{\mathrm{p} 1}$ of the integral in Eq. (17) is consequently larger. For example, for $a_{\mathrm{p}}=100, P_{\operatorname{det}}^{\mathrm{S}}\left(a_{\mathrm{p}}\right)$ is a factor of 2 and 10 larger for $\xi=3$ than for $\xi=6$ and $\xi=43$, respectively. We note, for reference, that the Moon has $\xi=6$ and the satellite Io of Jupiter would have $\xi=43$ if the planet were located at 1 AU (i.e., $a_{\mathrm{p}}=215$ ) from the Sun. Figure 1a also shows that $P_{\text {det }}^{\mathrm{S}}\left(a_{\mathrm{p}}\right)$ is not defined at small $a_{\mathrm{p}}$ for large $\xi$ because for a satellite to subsist, $a_{\mathrm{p}}$ must be larger than $a_{\mathrm{R}} \xi M_{\mathrm{p}}^{-1 / 3}$ (Sect. 2.1).

Figure $1 \mathrm{~b}$ shows the effect on $P_{\text {det }}^{\mathrm{S}}\left(a_{\mathrm{p}}\right)$ of changing the planet mass $M_{\mathrm{p}}$ for fixed $\xi=6$ and $i_{\mathrm{ps}}=10^{\circ}$, while Fig. 1c shows the effect of changing $i_{\mathrm{ps}}$ for fixed $M_{\mathrm{p}}=M_{\mathrm{J}}$ and $\xi=6$. The fact that the detection probability appears to increase with the planet mass results from our parameterization of $a_{\mathrm{s}}=\xi^{-1} M_{\mathrm{p}}^{1 / 3} a_{\mathrm{p}}$, which for fixed $\xi$ and $a_{\mathrm{p}}$ implies that $a_{\mathrm{s}}$ increases with $M_{\mathrm{p}}$. In fact, massive planets have larger Hill spheres, and hence, they may have more distant satellites. The case $M_{\mathrm{p}}=0.15 M_{\mathrm{J}}\left(\approx 50 M_{\mathrm{E}}\right)$ in Fig. 1b corresponds to a planet with $r_{\mathrm{p}} \approx 2.5 r_{\mathrm{E}}$ (where $M_{\mathrm{E}}$ and $r_{\mathrm{E}}$ are the Earth mass and radius), which according to Guillot et al. (1996) is the maximum radius for a terrestrial planet. As expected, Fig. 1c shows that $P_{\text {det }}^{\mathrm{S}}\left(a_{\mathrm{p}}\right)$

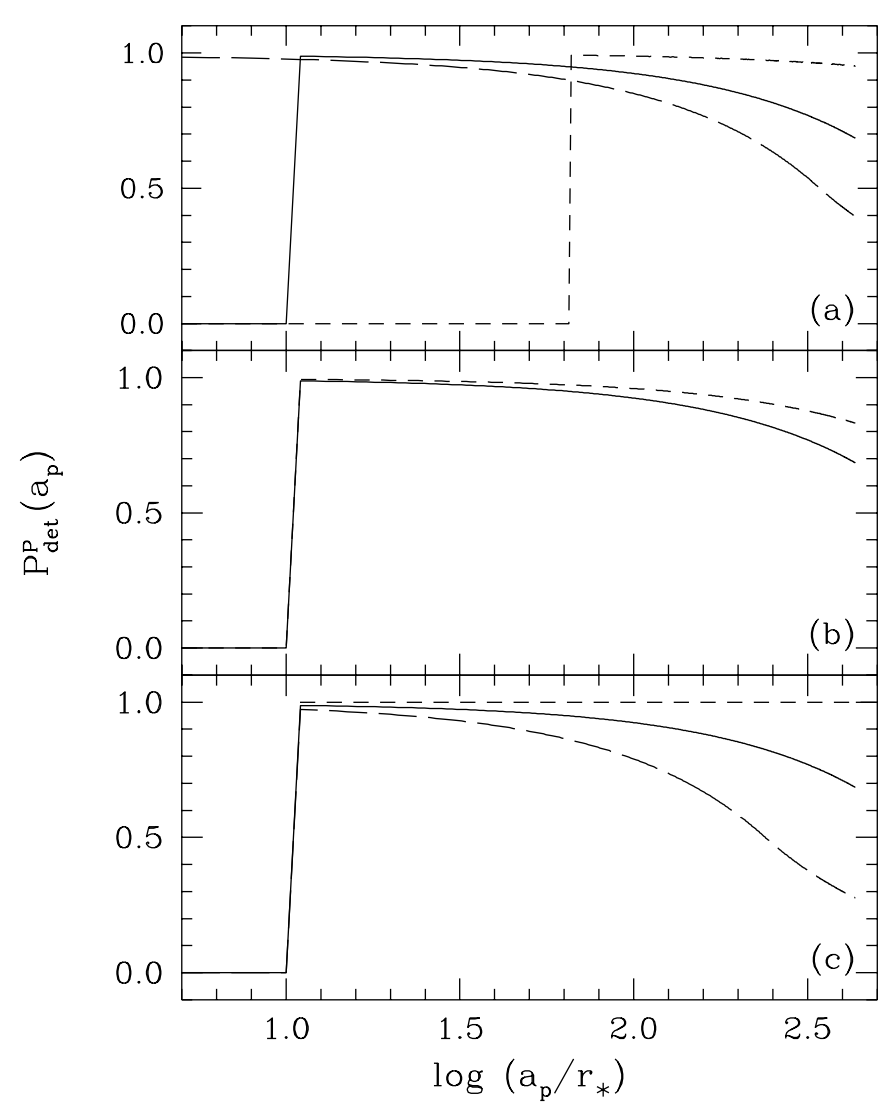

Fig. 3. Probability $P_{\text {det }}^{\mathrm{P}}\left(a_{\mathrm{p}}\right)$ of detecting a satellite transit during a planetary period, when the parent planet also transits over the star. The different curves in the three panels correspond to the same model assumptions as in Fig. 1

is higher when the angle between the planet and satellite orbital planes is larger, since again the upper limit $i_{\mathrm{p} 1}$ of the integral in Eq. (17) is consequently larger. For example, for $a_{\mathrm{p}}=100, P_{\mathrm{det}}^{\mathrm{S}}\left(a_{\mathrm{p}}\right)$ is a factor of 2.8 and 50 larger for $i_{\mathrm{ps}}=30^{\circ}$ than for $i_{\mathrm{ps}}=10^{\circ}$ and $i_{\mathrm{ps}}=0^{\circ}$, respectively.

The detection probability of a pure satellite transit will be increased with respect to the predictions of Fig. 1 if the parent planet orbits more than once during the period of observations. In Fig. 2 we show $P_{\text {det }}^{\mathrm{S}}\left(a_{\mathrm{p}}\right)$ assuming a period of observations of 3 years, for the same satellite and planet parameters as in Fig. 1b. Since the planet period scales as $a_{\mathrm{p}}^{3 / 2}$, independent of mass, the relative increase in $P_{\text {det }}^{\mathrm{S}}\left(a_{\mathrm{p}}\right)$ at fixed $a_{\mathrm{p}}$ with respect to Fig. $1 \mathrm{~b}\left(n_{\mathrm{t}}=1\right)$ is the same for satellites of $M_{\mathrm{p}}=M_{\mathrm{J}}$ and $M_{\mathrm{p}}=0.15 M_{\mathrm{J}}$ planets. The increase in $P_{\text {det }}^{\mathrm{S}}\left(a_{\mathrm{p}}\right)$ is slightly larger at small $a_{\mathrm{p}}$, corresponding to short periods, than at large $a_{\mathrm{p}}$. For example, $P_{\mathrm{det}}^{\mathrm{S}}\left(a_{\mathrm{p}}\right)$ is a factor of 3.9 larger at $a_{\mathrm{p}}=30$ and a factor of 3.6 larger at $a_{\mathrm{p}}=100$ than in the case $n_{\mathrm{t}}=1$. This result applies to all the curves in Fig. 1 . For comparison, we also show in Fig. 2 the probability $a_{\mathrm{p}}^{-1}$ of detecting a planet. When $a_{\mathrm{p}}$ is large, therefore, the probability to detect a planet is only slightly larger than that of detecting a satellite without a planet. 
We now turn to the case in which the planet is known to transit. Figures $3 \mathrm{a}, \mathrm{b}$ and $\mathrm{c}$ show $P_{\mathrm{det}}^{\mathrm{P}}\left(a_{\mathrm{p}}\right)$ for the same planet and satellite parameters as in Fig. 1 . In all cases the probability to detect a satellite during the transit of the planet is very close to unity. The main features on the curves may be understood from the above discussion of Fig. 1, after operating a multiplication by $a_{\mathrm{p}}$. Unlike $P_{\text {det }}^{\mathrm{S}}\left(a_{\mathrm{p}}\right)$, however, $P_{\mathrm{det}}^{\mathrm{P}}\left(a_{\mathrm{p}}\right)$ increases for combinations of parameters leading to smaller satellite orbital radii and inclinations, since the planet itself transits.

\subsection{Examples of possible lightcurves}

We now compute examples of stellar lightcurves expected during the transit of a planet and satellite system along the line of sight. Two types of lightcurves are expected in the case in which both the planet and the satellite transit in front of the star, depending on whether the orbital period of the satellite, $T_{\mathrm{S}}$, is shorter or longer than the duration of the planetary transit, $D_{\mathrm{T}}$. If $T_{\mathrm{S}} \ll D_{\mathrm{T}}$, planetsatellite transits may occur during the transit of the planet if the line-of-sight inclination of the satellite orbital plane is such that $i_{\mathrm{s}}<r_{\mathrm{p}} / a_{\mathrm{s}}$, where $r_{\mathrm{p}}$ is the planet radius (see Sect. 2.1). By planet-satellite transit we refer to both the transit of the satellite over the planet and the transit (occultation) of the planet over the satellite. Therefore, if $T_{\mathrm{S}} \ll D_{\mathrm{T}}$ and $i_{\mathrm{s}}<r_{\mathrm{p}} / a_{\mathrm{s}}$, there are at least two planetsatellite transits during a satellite orbit, at the two satellite conjunctions. If, on the other hand, $T_{\mathrm{S}} \gg D_{\mathrm{T}}$, a planetsatellite transit may or may not occur during a planetary transit for $i_{\mathrm{s}}<r_{\mathrm{p}} / a_{\mathrm{s}}$, depending on whether the satellite happens to pass through one of the two favorable conjunctions on its orbit in the time interval $D_{\mathrm{T}}$.

The upmost interest of planet-satellite transits is that they produce a relative increase of the apparent stellar luminosity, by an amount equal to the square of the satellite radius, $r_{\mathrm{s}}^{2}$ (see Eq. (1)), during the transit of the planet and satellite system in front of the star. Hence, planetsatellite transits can help to constrain observationally not only the presence, but also the period and orbital radius of the satellites of extrasolar planets. It should be noted that, since the condition for a planetary transit is $i_{\mathrm{p}}<a_{\mathrm{p}}^{-1} \ll 1$, the above condition on $i_{\mathrm{s}}$ is almost equivalent to the condition $i_{\mathrm{ps}}=i_{\mathrm{s}}-i_{\mathrm{p}} \approx i_{\mathrm{s}}<r_{\mathrm{p}} / a_{\mathrm{s}}$. Also, we do not show here examples of lightcurves for the case in which the satellite alone transits in front of the star because these are similar to classical lightcurves of planetary transits (see Fig. 4b).

The condition for observing at least one planet-satellite transit, if $i_{\mathrm{s}}<r_{\mathrm{p}} / a_{\mathrm{s}}$, can be written as

$T_{\mathrm{R}}<T_{\mathrm{S}}<2 \times D_{\mathrm{T}}$,

where $T_{\mathrm{R}}$ is the period corresponding to the Roche limit. The factor of 2 in the above expression follows from the two conjunctions on a satellite orbit that can lead to a planet-satellite transit during a satellite period. The duration of the planetary transit can be rewritten in terms of the planet orbital radius and period as $D_{\mathrm{T}}=T_{\mathrm{P}}\left(\pi a_{\mathrm{p}}\right)^{-1}$. Applying the third Kepler law to reexpress $a_{\mathrm{p}}$ in terms of $T_{\mathrm{P}}$, we obtain, for the condition on $T_{\mathrm{S}}$ for at least one planet-satellite transit,

$T_{\mathrm{R}}<T_{\mathrm{S}}<0.15 T_{\mathrm{P}}^{1 / 3}$

where units are days. The Roche period depends only on the satellite density and is $T_{\mathrm{R}}=0.23$ day for a terrestrial satellite with $\rho=5 \mathrm{~g} \mathrm{~cm}^{-3}$. Thus, the satellite period must be very short, at least an order of magnitude shorter than the planet period, for planet-satellite transits to occur. By virtue of the third Kepler law this also implies that satellite orbital radius, $a_{\mathrm{s}}$, must be small, and hence, that the condition $i_{\mathrm{s}}<r_{\mathrm{p}} / a_{\mathrm{s}}$ is also likely to apply. Another implication of Eq. (23) is that the period of the planet iself must be larger than about $300\left(T_{\mathrm{R}} / \text { days }\right)^{3}$, or roughly 4 days for a terrestrial satellite, in order for a planet-satellite transit to occur.

The actual number of planet-satellite transits that will occur during a planetary transit is $N_{\mathrm{PS}}=2 D_{\mathrm{T}} / T_{\mathrm{S}}$. Adopting the same parameterization as before of the satellite orbital radius, $a_{\mathrm{s}} \propto \xi^{-1} a_{\mathrm{p}}$ with $1 \leq \xi \leq a_{\mathrm{H}} / a_{\mathrm{R}}$, this yields $N_{\mathrm{PS}}=0.15 \xi^{3 / 2} T_{\mathrm{P}}^{-2 / 3}$. Note that since the satellite period at the Hill radius is equal to the planet period, one also has $1 \leq \xi \leq\left(T_{\mathrm{P}} / T_{\mathrm{R}}\right)^{2 / 3}$. Therefore, $\xi$ must be large, implying again that the satellite orbital radius must be small compared to the planet orbital radius, in order for $N_{\text {PS }}$ to be at least unity. For example Jupiter satellite Io has $\xi \approx 215$ and would transit Jupiter about twice during a transit of the planet in front of the Sun. On the other hand, if the Jupiter-Io system were located at only $1 \mathrm{AU}$ from the Sun, the satellite would have only $\xi \approx 43$ and the corresponding $N_{\mathrm{PS}}$ would be of order unity. In this example, however, Io would be too small by a factor of about 4 in radius to produce a relative increase of the stellar luminosity that could be detected directly with a photometric accuracy of $10^{-4}$ (but see Sect. 3.3).

Figure 4a shows the transit lightcurve of a planet with $r_{\mathrm{p}}=r_{\mathrm{J}}$ and $T_{\mathrm{P}}=50$ days and a satellite with $r_{\mathrm{s}}=2.5 r_{\mathrm{E}}$ and $T_{\mathrm{S}}=0.5$ days. Here, $r_{\mathrm{J}}$ and $r_{\mathrm{E}}$ are the Jupiter and Earth radii, respectively. The stellar flux shows a marked but moderate decrease at $t=-5 \mathrm{hr}$, as the satellite first transits in front of the star. When the planet also starts to transit, at $t=-3.5 \mathrm{hr}$, the stellar flux drops more abruptly. Soon after, at $t=-1 \mathrm{hr}$, the relative flux maximum produced by the planet-satellite transit appears clearly. Then, the stellar flux sharply increases as the planet leaves the star, and the satellite, which now follows the planet, continues to moderately occult the star from about $t=3.5$ to $4.5 \mathrm{hr}$. The curvature of the lightcurve is a consequence of the star limb-darkening, the flux minimum corresponding to the point at which the planet is closest to the stellar center. Also shown by crosses in Fig. 4a are the results of simulated 10 min exposure observations with a poisson noise of $10^{-4}$, typical of the photometric accuracy expected with the satellite COROT for $10^{\text {th }}$ magnitude 


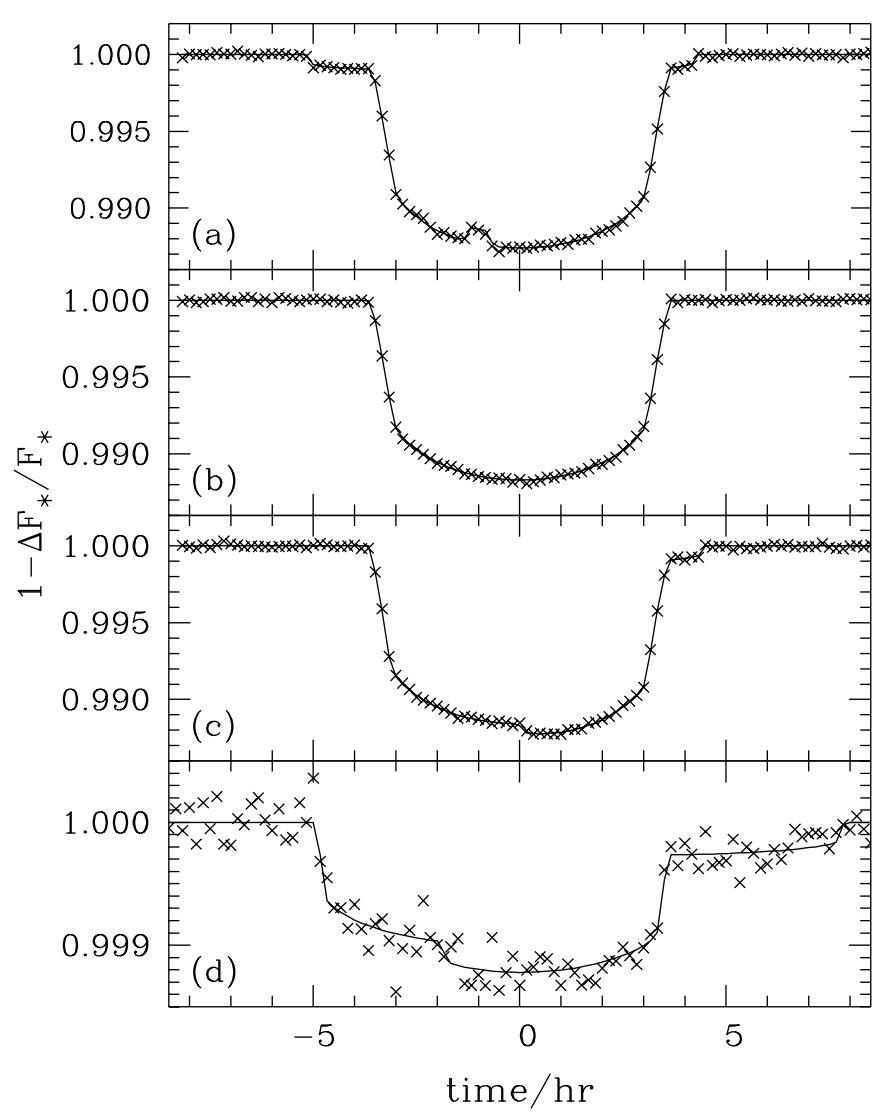

Fig. 4. Examples of transit lightcurves (see text for interpretation). a) For a planet with $r_{\mathrm{p}}=r_{\mathrm{J}}$ and $T_{\mathrm{P}}=50$ days and a satellite with $r_{\mathrm{s}}=2.5 r_{\mathrm{E}}$ and $T_{\mathrm{S}}=0.5$ days. b) For a planet with $r_{\mathrm{p}}=r_{\mathrm{J}}$ and $T_{\mathrm{P}}=50$ days, alone. c) For a planet with $r_{\mathrm{p}}=r_{\mathrm{J}}$ and $T_{\mathrm{P}}=50$ days and a satellite with $r_{\mathrm{s}}=2.5 r_{\mathrm{E}}$ and $T_{\mathrm{S}}=1.5$ days. d) For a smaller planet with $r_{\mathrm{p}}=2.5 r_{\mathrm{E}}$ and $T_{\mathrm{P}}=100$ days and a satellite with $r_{\mathrm{s}}=1.5 r_{\mathrm{E}}$ and $T_{\mathrm{S}}=2$ days. In all panels the solid line is the model lightcurve, and the crosses show the results of simulated 10 min exposure observations with a poisson noise of $10^{-4}$

stars. All feature of the lightcurve are mapped faithfully. In particular, the planet-satellite transit is detected with a signal-to-noise ratio of order 10. For reference, Fig. $4 \mathrm{~b}$ shows the transit lightcurve of the same planet but without a satellite.

If the satellite period does not satisfy condition (23) for at least one planet-satellite transit, the probability for the satellite to pass through one of the two favorable conjunctions that will produce a transit during the time interval $D_{\mathrm{T}}$ is simply given by $N_{\mathrm{PS}}$. As shown above, the probability will be highest for satellites with smallest orbital radii. The Moon, for example, has $T_{\mathrm{S}} \gg 0.15 T_{\mathrm{P}}^{1 / 3}$ and $\xi \approx 6$, and it would have a probability of only 0.04 to be observed in a transit over or behind the Earth during a transit of the planet over the Sun.

Figure 4c shows the transit lightcurve of the same planet as in Fig. 4a, but with a satellite with $r_{\mathrm{s}}=2.5 r_{\mathrm{E}}$ and $T_{\mathrm{S}}=1.5$ days, which does not satisfy condition (23).
The abrupt drop of the stellar flux at the beginning of the transit is caused by the entry of the planet. The pronounced, but more moderate drop at $t \approx 0 \mathrm{hr}$ is caused by the subsequent entry of the satellite. Then, the planet first leaves the star causing the flux to increase sharply. The satellite continues to occult the star from about $t=3.5$ to $4.5 \mathrm{hr}$ until it finally also leaves, and the stellar flux retrieves its original value. Again, the crosses show the results of simulated 10 min exposure observations with a poisson noise of $10^{-4}$. For comparison, we show in Fig. $4 \mathrm{~d}$ the transit lightcurve for a smaller planet with $r_{\mathrm{p}}=2.5 r_{\mathrm{E}}$ and $T_{\mathrm{P}}=100$ days and a satellite with $r_{\mathrm{s}}=1.5 r_{\mathrm{E}}$ and $T_{\mathrm{S}}=2$ days. The characteristic signatures of the entry and exit of first the planet and then the satellite are similar to those in Fig. 4c. Although the signal-to-noise level is about 4 times smaller than in Fig. 4c, the satellite and planet transits are still detected unambiguously.

\section{Indirect detection by timing of the planet transits}

If a satellite is not extended enough to produce a detectable signal in the stellar lightcurve, it may still be detected indirectly through the associated rotation of the planet around the barycenter of the planet-satellite system. This requires that a planetary transit be observed at least 3 times, as the effect of the rotation will be a periodical time shift of the lightcurve minima induced by the planet transits. We now estimate the expected time shift in the simple case where the satellite and planet orbital planes are aligned along the line of $\operatorname{sight}\left(i_{\mathrm{ps}} \approx i_{\mathrm{p}} \approx 0\right)$. In this case, the projected diameter of the planet orbit around the barycenter of the planet-satellite system is $2 a_{\mathrm{s}} M_{\mathrm{s}} M_{\mathrm{p}}^{-1}$, where $M_{\mathrm{s}}$ is the satellite mass. Therefore, the expected time shift between transits will be

$\Delta t \sim 2 a_{\mathrm{s}} M_{\mathrm{s}} M_{\mathrm{p}}^{-1} \times T_{\mathrm{p}}\left(2 \pi a_{\mathrm{p}}\right)^{-1}$.

Measurements of $\Delta t$, in addition to revealing the presence of a satellite, provide also an estimate of the product of its mass and orbital radius, $a_{\mathrm{s}} M_{\mathrm{s}}$. The minimum detectable $a_{\mathrm{s}} M_{\mathrm{S}}$ is determined by the the minimum measurable time shift, and hence, by the accuracy of the timing of lightcurve minima. If $\delta t_{\text {obs }}$ denotes the sampling time, i.e., the duration of each consecutive exposure, then we can expect to be sensitive to the presence of satellites with $M_{\mathrm{s}} a_{\mathrm{s}} \sim M_{\mathrm{p}} a_{\mathrm{p}} \pi \delta t_{\mathrm{obs}} / T_{\mathrm{p}}$.

We have estimated the minimum sampling time required to determine the position of a lightcurve minimum with a timing accuracy of $\delta t_{\text {obs }}$. We simulated observed lightcurves using different values of $\delta t_{\text {obs }}$ and considering poisson noise only, which we cross-correlated with the corresponding input model lightcurves. We find that the minimum $\delta t_{\text {obs }}$ required corresponds to the exposure time needed to detect the depth of the planetary transit minimum at twice the photon noise level, i.e., $\left|\Delta F_{*}\right|>2 \sigma$ (see Eq. (1)). As an example, the minimum $\delta t_{\text {obs }}$ would 
be about $0.6 \mathrm{~s}$ to time the transit of a Jupiter-like planet over a solar-type star of magnitude 10 with a telescope like the one onboard COROT, while it would be $\delta t_{\mathrm{obs}} \approx 4 \mathrm{~min}$ to time the transit of a planet with radius $2 r_{\mathrm{E}}$ over the same star. According to Eq. (25), one could then infer the presence of satellites with the mass of Io or with a mass a tenth that of the Earth, respectively, in these two cases.

As a final note, we mention some examples of the possible implications of our results for future observations with COROT. Transits of planets and satellites larger than about $2 r_{\mathrm{E}}$ are expected to be detectable with COROT (see Sect. 2). The results of Sect. 3 and Sect. 4 then indicate that, if present, planets photometrically detectable and with orbital radii $a_{\mathrm{p}}=0.1,0.2$, and $0.3 \mathrm{AU}$ should have probabilities $4.6 \%, 2.3 \%$, and $1.5 \%$, respectively, to produce observable transits. Also, if these planets have satellites that are themselves photometrically detectable, the probability to detect the satellites is found to be $\sim 100 \%$. The main reason for this is that since the planets under consideration have small orbital radii, the inclination of the orbital plane of a satellite is expected to be close to that of the parent planet. A satellite will also induce time shifts between successive planet transits because of the rotation of the planet around the barycenter of the planet-satellite system. Via this effect, with COROT it will be possible to detect or exclude the presence of satellites much smaller than those photometrically detectable (i.e., with $r_{\mathrm{s}} \sim 0.3 r_{\mathrm{E}}$ around a Jupiter-like planet for a sampling time $\left.\delta t_{\mathrm{obs}} \approx 0.6 \mathrm{~s}\right)$. COROT, therefore, will set important constraints on the existence of satellites around detectable planets.
Acknowledgements. P.S. is an ESA fellow. We thank the referee for helpful comments.

\section{References}

Allen C.W., 1973, Astrophysical Quantities. London: Asthlone Borucki W.J., Koch D.G., Dunham E.W., et al., 1997, in: The Kepler Mission: A Mission To Determine The Frequency of Inner Planets Near The Habitable Zone of A Wide Range of Stars, ASP Conf. Ser. (in press)

Burns J.A., 1986, in Satellites, Burns J.A. \& Matthews (eds.) Butler P., Marcy G., Williams E., et al., 1997, ApJ 474, L115

Cochran W.D., Hatzes A.P., Butler R.P., et al., 1997, ApJ 483, 457

Frölich C., 1987, JGR 92, 796

Guillot T., Burrows A., Hubbard W.B., et al., 1996, ApJ 459, L35

Koch D.G., Borucki W.J., Cullers K., et al., 1996, JGR-Planets 101, 9297-9302

Marcy G.W., Butler R.P., 1996, ApJ 464, L147

Mayor M., Queloz D., 1995, Nat 378, 355

Struve O., 1952, The Observatory 72, 199

Schneider J., Doyle L.R., 1995, Earth Moon, Planets 71, 153

Schneider J., Appourchoux T., Auvergne M., Baglin A., et al., 1997, in Proceedings of the First NASA Origins Conference, M. Shull (ed.) (in press)

Willson R.C., Hudson H.S., 1991, Nat 351, 42 